\title{
CTLA-4 is expressed by human monocyte-derived dendritic cells and regulates their functions
}

\author{
Stefania Laurent ${ }^{\mathrm{a}}$, Paolo Carrega ${ }^{\mathrm{b}}$, Daniele Saverino ${ }^{\mathrm{c}}$, Patrizia Piccioli ${ }^{\mathrm{d}}$, Marta Camoriano ${ }^{\mathrm{e}}$, \\ Anna Morabito ${ }^{\mathrm{e}}$, Beatrice Dozin ${ }^{\mathrm{f}}$, Vincenzo Fontana ${ }^{\mathrm{g}}$, Rita Simone ${ }^{\mathrm{c}}$, Lorenzo Mortara ${ }^{\mathrm{h}}$, \\ Maria Cristina Mingari ${ }^{\mathrm{i}, \mathrm{j}}$, Guido Ferlazzo ${ }^{\mathrm{k}}$, Maria Pia Pistillo ${ }^{\mathrm{e}, *}$ \\ a Department of Hematology and Oncology, University of Genoa, Genoa, Italy \\ ${ }^{\mathrm{b}} \mathrm{G}$. Gaslini Institute, Genoa, Italy \\ ${ }^{c}$ Department of Experimental Medicine, University of Genoa, Genoa, Italy \\ ${ }^{\mathrm{d}}$ Medical Oncology C, National Institute for Cancer Research, Genoa, Italy \\ e Breast Cancer Laboratory of Tumour Genetics Unit, National Institute for Cancer Research, Genoa, Italy \\ ${ }^{\mathrm{f}}$ Clinical Epidemiology Unit, National Institute for Cancer Research, Genoa, Italy \\ $\mathrm{g}$ Unit of Epidemiology and Biostatistics, National Institute for Cancer Research, Genoa, Italy \\ ${ }^{\mathrm{h}}$ Department of Clinical and Biological Sciences, School of Medicine, University of Insubria, Varese, Italy \\ i Department of Oncology, Biology and Genetics, University of Genoa, Genoa, Italy \\ ${ }^{\mathrm{j}}$ Department of Translational Oncology, National Institute for Cancer Research, Genoa, Italy \\ ${ }^{\mathrm{k}}$ Department of Human Pathology, School of Medicine, University of Messina, Messina, Italy
}

\section{A R R I I C L E I I N F O}

\section{Article history:}

Received 7 April 2010

Accepted 12 July 2010

Available online 2 August 2010

\section{Keywords:}

CTLA-4

DC

Cytokine secretion

T-cell proliferation

\begin{abstract}
A B S T R A C T
Cytotoxic T lymphocyte antigen-4 (CTLA-4) is the major negative regulator of T-cell responses, although growing evidence supports its wider role as an immune attenuator that may also act in other cell lineages. Here, we have analyzed the expression of CTLA-4 in human monocytes and monocyte-derived dendritic cells (DCs), and the effect of its engagement on cytokine production and T-cell stimulatory activity by mature DCs. CTLA-4 was highly expressed on freshly isolated monocytes, then down-modulated upon differentiation toward immature DCs (iDCs) and it was markedly upregulated on mature DCs obtained with different stimulations (lipopolysaccharides [LPS], Poly:IC, cytokines). In line with the functional role of CTLA-4 in T cells, treatment of mDCs with an agonistic anti-CTLA-4 mAb significantly enhanced secretion of regulatory interleukin (IL)-10 but reduced secretion of IL-8/IL-12 pro-inflammatory cytokines, as well as autologous $\mathrm{CD}^{+}$T-cell proliferation in response to stimulation with recall antigen purified protein derivative (PPD)

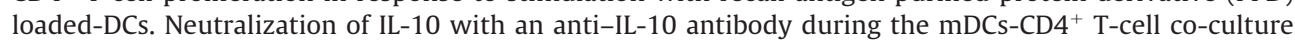
partially restored the ability of anti-CTLA-4-treated mDCs to stimulate T-cell proliferation in response to PPD. Taken together, our data provide the first evidence that CTLA-4 receptor is expressed by human monocyte-derived mDCs upon their full activation and that it exerts immune modulatory effects.
\end{abstract}

(C) 2010 American Society for Histocompatibility and Immunogenetics. Published by Elsevier Inc. All rights

reserved.

\section{Introduction}

Cytotoxic T lymphocyte antigen-4 (CTLA-4; CD152) is one of the most extensively studied inhibitory co-receptor within the T-cell-mediated immune response. Its expression was initially restricted to activated T lymphocytes [1-3] and subsequently extended to a subset of $\mathrm{T}$ cells with immunoregulatory properties (regulatory T cells [Tregs]) [4]. CTLA-4 expression has also been documented in a variety of non-T cells, either normal or neoplastic, including activated B cells [5], monocytes [6], placental fibroblasts [7], muscle cells [8], leukemic cells [9], and breast and melanoma tumor cells $[10,11]$. In these cells, depending on the cell type and/or

\footnotetext{
* Corresponding author.

E-mail address: mariapia.pistillo@istge.it (M.P. Pistillo).

S.L. and P.C. contributed equally to this work.
}

activation status, CTLA- 4 is expressed at different levels, both on the surface and in the cytoplasm. Although numerous reports have addressed the CTLA-4 inhibitory role in T cells, its functional significance in different cell types so far has not been widely explored.

CTLA-4 inhibitory function in T cells mainly occurs upon engagement with the B7 ligands (CD80/CD86) [12] expressed on antigen presenting cells, which results in the inhibition of both cytokine production and T-cell proliferation $[13,14]$. Several mechanisms have been proposed to explain CTLA-4 inhibitory function, including indirect effects such as competition for CD80/CD86 binding by the related molecule $\mathrm{CD} 28$, and/or direct effects such as binding to signaling molecules (PI3K, SHP2, PP2A), interference with TCR activation pathway and lipid rafts formation, inhibition of cyclin D3, cyclin-dependent kinases (cdk4/cdk6), and nuclear transcription factors (nuclear factor- $-\mathrm{B}[\mathrm{NF}-\kappa \mathrm{B}]$, NF-AT, AP-1), as re- 
viewed by Teft et al. [15]. The binding of CTLA- 4 with anti-CTLA-4 $\mathrm{mAbs}$ or CD80 ligand was shown to inhibit IL-2 production and proliferation of primary CD4 ${ }^{+}$T cells $[14,16]$ and IL-4 and IFN- $\gamma$ production of T-cell clones [17]. In addition, the inhibitory signal transduced by CTLA- 4 binding is able to increase IL- 10 and TGF- $\beta$, showing an additional effect in regulating this pathway [18].

Besides inhibiting directly T-cell activation, CTLA-4, either in its membrane-bound or soluble form, may also down-modulate Tcell-mediated immune response through the interaction with B7 ligands expressed by mature dendritic cells (DCs). In fact, as suggested by studies in animal models of transplant tolerance [19,20], B7 engagement by a soluble CTLA-4 fusion protein (CTLA-4-Ig) or CTLA-4 expressed by Tregs may induce activation of the indoleamine 2,3-dioxygenase (IDO) enzyme and initiate tryptophan catabolism in DCs, thus reducing T-cell proliferation and survival [19,21].

Therefore, CTLA-4 may exert its immunoregulatory role through a bidirectional signaling between CTLA-4 on T cells and B7 on DCs that results in attenuation of ongoing immune response and maintenance of $\mathrm{T}$-cell homeostasis. Although previous reports have shown constitutive or inducible expression of B7 molecules also on resting or activated human and murine T cells [22,23], suggesting a possible T-T interaction, expression of CTLA- 4 by human DCs has so far not been demonstrated.

In this study, we report that CTLA-4 expression can be induced on matured human monocyte-derived DCs. We demonstrate that treatment of mDCs with an agonistic anti-CTLA-4 antibody has functional effects as it modulates cytokine secretion and decreases $\mathrm{mDC}$-induced antigen-specific $\mathrm{CD}^{+}{ }^{+} \mathrm{T}$-cell proliferation, the latter being caused at least partially by an increase in IL-10 production upon CTLA-4 engagement on mDCs.

\section{Subjects and methods}

\subsection{Isolation of peripheral blood cells and $D C$ generation}

Human peripheral heparinized blood samples were obtained from voluntary healthy donors upon informed consent, according to institutional procedures and the Declaration of Helsinki. Peripheral blood mononuclear cells (PBMCs) were purified by density gradient centrifugation (Ficoll; Biochrom, Berlin, Germany) and subjected to immunomagnetic positive selection with an antibody against monocytes (CD14; Miltenyi Biotec, Auburn, CA). Monocytes were $>98 \%$ pure as determined by anti-CD14 mAb staining. Monocytederived DCs were generated by culturing CD14 ${ }^{+}$cells for 6 days in 24-well plates $\left(5 \times 10^{5}\right.$ cells $/ \mathrm{ml}$ ) with RPMI 1640 (Cambrex Bio Science, Verviers, Belgium) supplemented with $10 \%$ heat-inactivated fetal calf serum (Cambrex) (complete medium). To induce immature DCs, recombinant human IL-4 and recombinant human GM-CSF (Euroclone, Milan, Italy) were added at days 0 and 2 at final concentrations of 25 and $20 \mathrm{ng} / \mathrm{ml}$, respectively.

On culture day 4 , half of the medium was replaced with fresh complete medium containing IL-4, GM-CSF, and different DCmaturation stimuli including Escherichia coli lipopolysaccharide (LPS; St. Louis, MO) at $100 \mathrm{ng} / \mathrm{ml}$, Polyl:C (Calbiochem, DBA, Milan, Italy) at $25 \mu \mathrm{g} / \mathrm{ml}$, or cocktail of cytokines, including IL-6, IL-1 $\beta$, TNF- $\alpha$ (all from Euroclone) and $\mathrm{PGE}_{2}$ (Sigma-Aldrich, Milan, Italy), at $10 \mathrm{ng} / \mathrm{ml}$ for 2 days, to stimulate complete mDCs. To study the effects of CTLA- 4 binding, iDC were cultured for 48 hours in the presence of the agonistic anti-CTLA-4 mAb 3D5 [5,18], kindly provided by A. Lanzavecchia (Institute for Research in Biomedicine, Bellinzona, Switzerland), coated on a plastic plate, and cells were collected for cell surface phenotyping and functional tests.

In addition, PBMCs were cultured in complete medium in the presence or not of phytohemaglutinin A (PHA; Life Technologies, Milan, Italy) at a final concentration of $2 \mu \mathrm{g} / \mathrm{ml}$ for 48 hours at $37^{\circ} \mathrm{C}$. $\mathrm{CD}^{+} \mathrm{T}$ lymphocytes were isolated from PBMCs by positive selec- tion with magnetic beads coated with mAb to CD4 (MACS, Miltenyi Biotec).

\subsection{Flow-cytometric analysis of cell surface markers and CTLA-4 expression}

Analysis of cell surface markers and CTLA- 4 on monocytes and dendritic cells was performed by direct immunofluorescence staining using the following murine monoclonal antibodies (mAbs): Peridinin-Chlorophyll-Protein Complex (PerCP)-conjugated antiHLA-DR (L247 clone), Phycoerythrin-conjugated anti-CD14 (M5E2 clone) (all from BD PharMingen, San Diego, CA), PE-conjugated anti-CD83 (HB15a clone) (Immunotech-Coulter, Fullerton, CA, USA), PE-conjugated anti-CD11c and Phycoerythrin Cyanin 5 (PC5)-conjugated anti-ILT3. For surface CTLA-4, the following two antibody reagents were used in independent experiments: the FITCconjugated anti-CTLA-4 mAb 48815 [24,25] and PE-conjugated goat anti-CTLA-4 polyclonal antibody (both from R\&D Systems, Minneapolis, MN).

Direct immunofluorescence was performed by staining, for $30 \mathrm{~min}$ utes at $4^{\circ} \mathrm{C}, 1 \times 10^{5}$ cells/sample with the fluorochrome-coniugated mAbs preparations containing $1 \mathrm{mg} / \mathrm{ml}$ purified human $\gamma$-globulin (human therapy grade, Biotest SRL, Milan, Italy) to prevent nonspecific binding to Fc-gamma receptor. Cells were washed twice in phosphate-buffered saline plus $2 \%$ fetal calf serum (FACS buffer) and analyzed by flow cytometry.

Analysis of intracellular CTLA-4 expression was performed by indirect immunofluorescence staining with the anti-CTLA-4 mAb 14D3 [26,27] (from eBioscence, San Diego, CA), for 30 minutes at $4^{\circ} \mathrm{C}, 4 \times 10^{5}$ cells/sample after cell fixation with $2 \%$ paraformaldehyde and permeabilization with $0.1 \%$ saponin. After washing in FACS buffer, a FITC-conjugated isotype-specific goat anti-mouse IgG secondary antibody (Southern Biotechnology, Birmingham, AL) was added and incubated for 30 minutes at $4^{\circ} \mathrm{C}$. After two final washes, cells were analyzed by flow cytometry. Negative controls included directly labeled and unlabeled isotype-matched irrelevant mAbs.

Flow-cytometric analysis was performed with a FACSCalibur cytometer (Becton Dickinson, San Jose, CA) using CellQuest software (Becton Dickinson). For gating on viable cells, we used TOPRO-3 iodide (Invitrogen, Milan, Italy), a membrane-impermeable DNA binding dye, added to samples ( $0.5 \mu \mathrm{M}$ final concentration), in addition to forward and side scatter characteristics of each cell type. A total of 10,000 gated events were acquired for each maturation and antibody condition. Results were expressed as mean ratio of relative fluorescence intensity (MRFI), calculated on the same cell fractions, as follows: mean fluorescence intensity of CTLA-4 staining/mean fluorescence intensity of irrelevant isotypematched mAb staining.

\subsection{RT-PCR analysis of CTLA-4 transcripts}

Total cellular RNA was extracted using RNeasy Mini Kit (Qiagen, Milan, Italy) following the manufacturer's instructions; residual DNA was removed by DNAse digestion. A 2- $\mu$ g quantity of total RNA was reverse transcribed into first-strand cDNA using the SuperScript II Reverse Transcriptase (Invitrogen). Amplification of CTLA- 4 transcripts was performed in a reaction mix, final volume of $20 \mu \mathrm{l}$, containing $100 \mathrm{ng}$ of cDNA, $0.1 \mathrm{mmol} / \mathrm{l} \mathrm{dNTPs}, 1$ U AmpliTaq Gold (Applied Biosystems, Roche, Milan, Italy), its specific buffer with $1.5 \mathrm{mmol} / 1 \mathrm{MgCL}_{2}$, and $0.25 \mu \mathrm{mol} / 1 \mathrm{lof}$ each primer (sense: 5' GACTCTACATCTGCAAGGT 3' and antisense: 5' CTCAGCTCTTGGAAATTG $3^{\prime}$ ).

Cycling conditions were 12 minutes at $95^{\circ} \mathrm{C}$ followed by 45 cycles of $94^{\circ} \mathrm{C}$ for 30 seconds, of $55^{\circ} \mathrm{C}$ for 30 seconds, $72^{\circ} \mathrm{C}$ for 30 seconds, and a final cycle at $72^{\circ} \mathrm{C}$ for 10 minutes. Polymerase chain reaction (PCR) products were separated by standard electrophore- 
sis on a $2.5 \%$ agarose gel containing ethidium bromide. Specificity of CTLA-4 PCR products was confirmed by direct sequencing analysis, in both directions, using an ABI-PRISM 3130 (Applied Biosystems, Foster City, CA).

\subsection{Cytokine production}

The agonistic anti-CTLA-4 mAb 3D5 (IgG1) was coated overnight at $4^{\circ} \mathrm{C}$ on flat-bottom plates and iDCs were plated at $1 \times 10^{6}$ cells $/ \mathrm{ml}$ on coated plates in the presence of LPS $(100 \mathrm{ng} / \mathrm{ml})$. Culture supernatants were collected after 24 and 48 hours of culture and all samples were tested in duplicate for the presence of IL-8/CXCL8, IL-10 and IL-12p70 cytokines by enzyme-linked immunosorbent assay (ELISA) following the manufacturer's instructions (Bender MedSystems, Milan, Italy). An anti-CD31 mAb was used as IgG1 isotype-matched control $\mathrm{mAb}$ as previously described [28].

\subsection{Apoptosis detection}

Immature DCs were cultured on plates coated with the antiCTLA-4 agonistic mAb or with the IgG1 isotype-matched control $\mathrm{mAb}$ in the presence of LPS $(100 \mathrm{ng} / \mathrm{ml})$. Mature DCs were collected after 24 and 48 hours to evaluate apoptosis by flow cytometry using annexin $\mathrm{V}$ binding and TO-PRO-3 uptake. Briefly, mDCs were collected, washed in annexin $\mathrm{V}$ binding buffer and resuspended at $5-10 \times 10^{5} \mathrm{mDCs} / \mathrm{ml}$ in $50 \mu \mathrm{l}$ of staining buffer containing $1 \mu \mathrm{l}$ of annexin V (MBL International, Woburn, MA) at $250 \mu \mathrm{g} / \mathrm{ml}, 5 \mu \mathrm{l}$ of TO-PRO-3 iodide (Invitrogen), $1 \mathrm{mmol}$ of solution, for 15 minutes in the dark at room temperature. Cells were analyzed by flow cytometry with a FACSCalibur cytometer (Becton Dickinson) using CellQuest software.

\subsection{CD4+ T lymphocyte proliferation assays}

Both $\mathrm{mDCs}$ and $\mathrm{CD} 4^{+} \mathrm{T}$ lymphocytes were obtained as described above. mDCs were pretreated with anti-CTLA- 4 and with IgG1 isotype control mAbs on 24-well, flat-bottom coated plates for 30 minutes at $4^{\circ} \mathrm{C}$, collected by pipetting, washed, and pulsed, at $3 \times 10^{5} / \mathrm{ml}$, with the recall antigen purified protein derivative (PPD) from Mycobacterium tuberculosis (Statens Serum Insitut, Copenhagen, Denmark) at a final concentration of $5 \mu \mathrm{g} / \mathrm{ml}$. Proliferative responses were measured by culturing $3 \times 10^{4}$ pretreated $\mathrm{mDCs}$ in the presence of $10^{5} \mathrm{CD}^{+}$autologous T lymphocytes in $0.2 \mathrm{ml}$ of complete medium, in 96-well, flat-bottom microtiter plates.

In another set of experiments, neutralizing anti-IL-10 mAb or control IgG2b isotype $\mathrm{mAb}$ were added to the co-cultures. All cultures were pulsed with $0.5 \mu \mathrm{Ci}$ of $\left[{ }^{3} \mathrm{H}\right]$ thymidine (Amersham Biosciences, Cologno Monzese, Italy) on day 4 and harvested 18 hours later. Dry filters with scintillation fluid were counted in a gamma counter (Beckman-Coulter). Counts represent mean values of triplicates.

\subsection{Statistical analyses}

Statistical analyses were performed to compare: (1) CTLA-4 expression levels on mDCs, iDCs and monocytes, (2) cytokine production by anti-CTLA-4-treated and isotype control-treated $\mathrm{mDCs}$, and (3) $\mathrm{CD} 4^{+} \mathrm{T}$-cell proliferation in co-culture experiments with mDCs treated with anti-CTLA-4 and anti-IL-10 mAbs or with the irrelevant control mAbs. All results are presented as mean \pm standard error of the mean. Differences between groups were evaluated by paired two-tailed Student's $t$ tests. All $p$ values (significance level $<0.05$ ) were further adjusted with Bonferroni correction for multiple comparisons and are indicated as " $p_{c}$." Statistical analyses were carried out using the SPSS package, version 17.0 for Windows (SPSS Inc., Chicago, IL).

\section{Results}

\subsection{CTLA-4 is expressed by human mature DCS}

To study CTLA-4 expression by human monocyte-derived DCs, peripheral blood monocytes were isolated by positive selection with anti-CD14 antibody-coated-magnetic beads (purity of CD14 ${ }^{+}$ cells was $>98 \%$ ), cultured with GM-CSF+IL-4 to differentiate into immature DCs and further stimulated with different maturation stimuli to generate mature DCs. CTLA-4 expression in the various cell populations was evaluated by immunofluorescence staining and flow cytometry with a panel of well-validated anti-CTLA-4 specific antibodies (see Subjects and methods).

CTLA-4 was found to be constitutively expressed on the surface of freshly isolated monocytes when tested immediately after collection from different donors, but disappeared within 24 hours of cultures in the presence of GM-CSF+IL-4 and remained below detectable levels up to 72 hours of culture. Matured $\mathrm{CD}^{2} 3^{+} \mathrm{DCs}$ obtained by in vitro stimulation of iDCs with different maturation stimuli, including LPS (bacterial), PolyI:C (viral), and cytokine cocktails (inflammatory), similarly resulted in a significant upregulation of CTLA-4 cell surface expression (Fig. 1A, Table 1). Remarkably, surface CTLA- 4 expression on $\mathrm{mDC}$ remained stable for at least 48 hours after different stimulations.

High levels of CTLA-4 were detected in the cytoplasm of monocytes, iDCs, and mDCs obtained with different stimuli, as shown by the flow-cytometric intracellular stainings (Fig. 1B). This pattern is consistent with the well known T-cell intracellular compartimentalization of CTLA-4 that is mainly localized in vesicles of the Golgi apparatus and is released to the cell surface during T-cell activation [29,30]. In agreement with previous reports [9,10], CTLA-4 expression was detected in the cytoplasm of both resting and PHAactivated PBMCs used as controls and on the surface of only PHA-activated PBMCs (Fig. 1C). As the different stimuli used resulted in similar CTLA-4 expression on activated DCs, we next analyzed CTLA-4 expression and its biologic effects only in LPSmatured DCs (LPS-mDCs). We compared the levels of CTLA-4 expression in LPS-mDCs with those in iDCs or monocytes derived from seven individual donors, by measuring the mean ratio of relative fluorescence intensity (MRFI). CTLA-4 expression resulted upregulated in LPS-mDCs, from six of seven donors examined (Table 1), showing MRFI values significantly higher than those in iDC samples $\left(p<0.001, p_{c}=0.002\right)$, but not significantly different from those in freshly isolated monocytes $\left(p=0.074, p_{c}=0.222\right)$. On the contrary, MRFI values in iDC samples were significantly lower than those in freshly isolated monocytes $\left(p=0.016, p_{c}=0.048\right)$.

Thus, our data demonstrate that upon in vitro maturation with different stimuli, human monocyte-derived mDCs upregulate CTLA-4 suggesting that this molecule can be expressed during DC maturation process.

\subsection{CTLA-4 TM transcript variant is expressed by human mature DCS}

CTLA-4 expression was confirmed at transcriptional level by RT-PCR analysis carried out with a set of primers that identifies two alternative variants of CTLA-4, namely the 348-bp fragment corresponding to the transcript variant 1 or CTLA-4 TM (GenBank accession number NM_005214) and the 238-bp fragment corresponding to the transcript variant 2 or CTLA-4delTM/soluble CTLA-4 (GenBank accession numbers NM_001037631). The transcriptional patterns shown (Fig. 1D) refer to representative freshly isolated human monocytes (MOs), iDCs, LPS-mDCs, and resting and PHA-activated PBMCs, the CTLA-4 expression pattern of which is shown by flow cytometry (Fig. 1A-C).

The results showed that freshly isolated human MOs, as well as iDCs, express both CTLA- 4 transcript variants with iDCs expressing lower levels of CTLA-4delTM compared with CTLA-4 TM transcript 

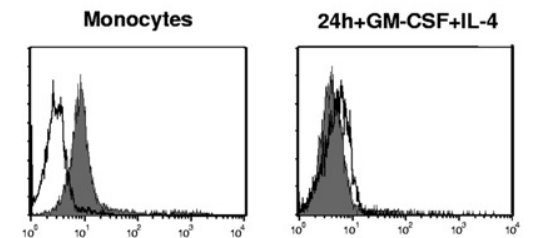

4 days culture (iDCs)

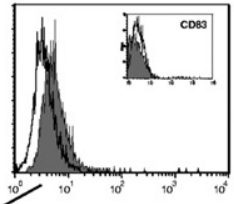

2 days of culture in the presence of stimuli
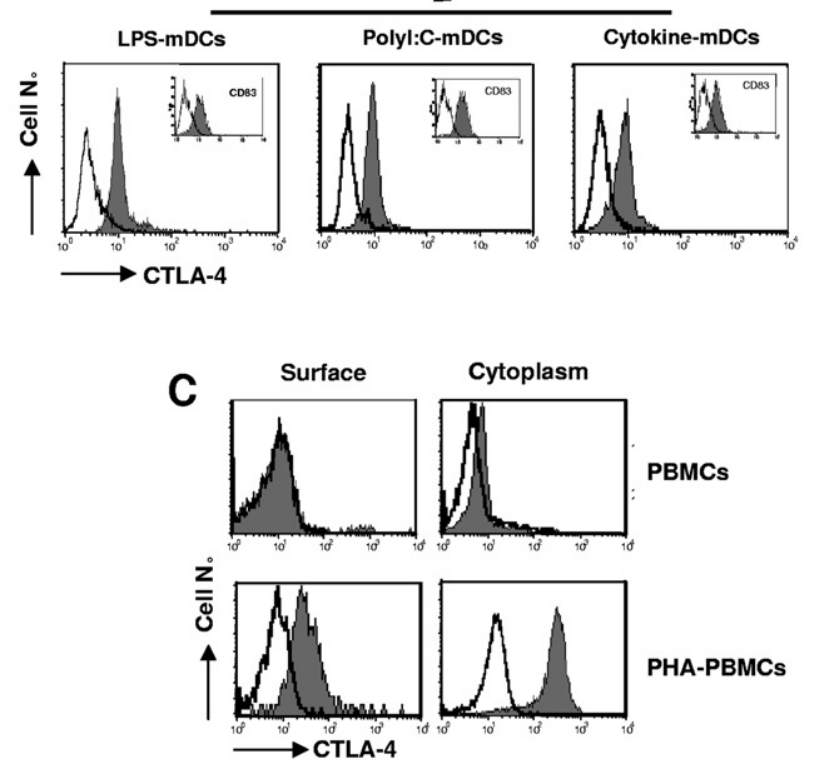

Isotype control

\section{Anti-CTLA-4 Ab}

B

Cytoplasmic CTLA-4

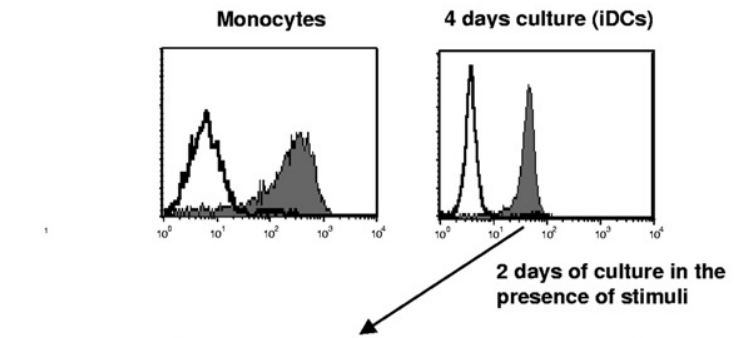

4 days culture (iDCs)

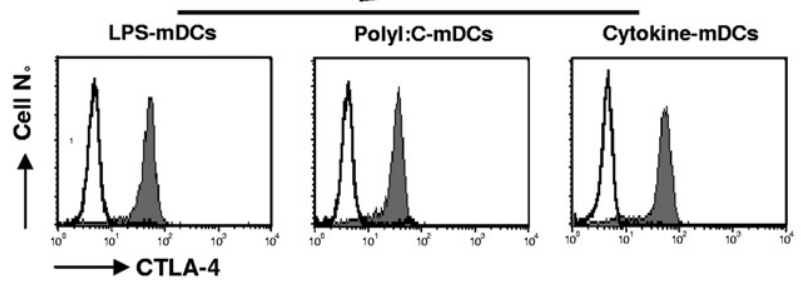

D CTLA-4 transcript variants

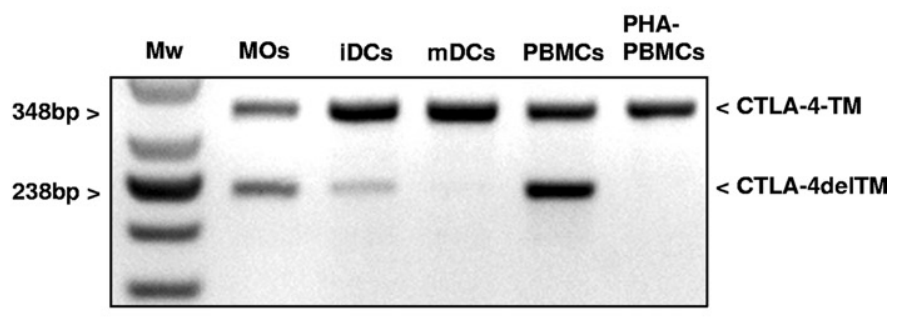

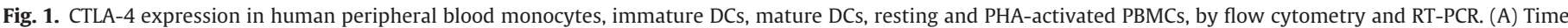

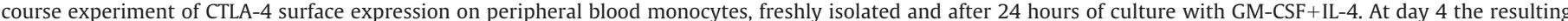

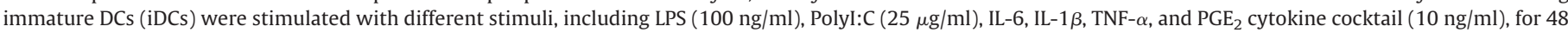

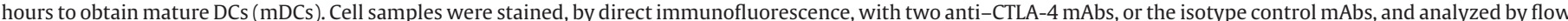

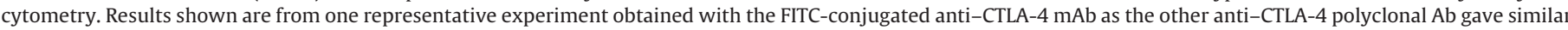

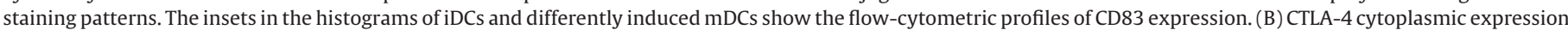

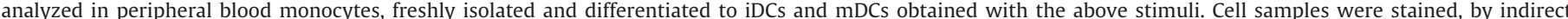

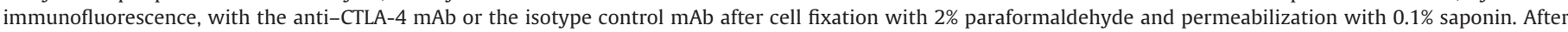

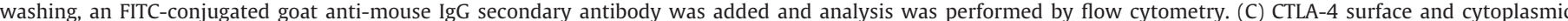

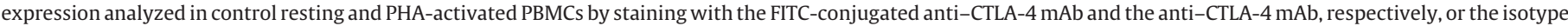

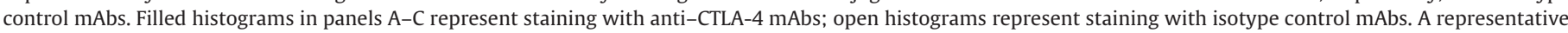

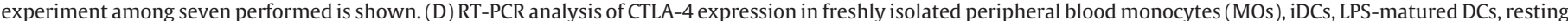

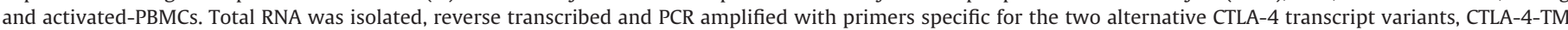

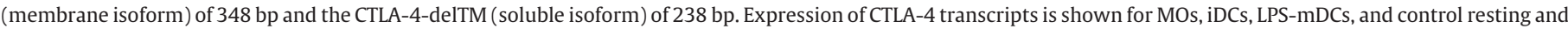
PHA-activated PBMCs from the representative experiment the CTLA-4 expression of which is shown by flow cytometry in panels A-C. Mw, molecular weight marker.

(Fig. 1D). LPS-mDCs showed similar expression of CTLA-4 TM but undetectable, or barely detectable, level of CTLA-4delTM transcript (Fig. 1D).

The expression pattern of these transcripts, which was also confirmed using another set of primers encompassing the $3^{\prime}$ of exon 1 (signal peptide) and exon 2 (nt243-nt343; data not shown), resembles the one previously described [10] for resting and PHAactivated PBMCs used here as positive controls of the CTLA-4 transcript variants (Fig. 1D).

\subsection{CTLA-4 engagement on human mature DCs downregulates IL-8} and IL-12 but upregulates IL-10 production

It has been reported that CTLA-4 plays a role in regulating cytokine production by both activated $\mathrm{T}$ [18] and $\mathrm{B}$ cells [5]. We thus investigated whether binding of CTLA- 4 expressed by mDCs with an agonistic anti-CTLA- 4 mAb exerts a similar effect on DCs. To this end, iDCs were cultured on plates coated with the anti-
CTLA-4 mAb 3D5 $[5,18]$ or with the IgG1 isotype-matched control $\mathrm{mAb}$ in the presence of LPS. Supernatants were collected after 24 and 48 hours of culture to measure the levels of the pro-inflammatory cytokines IL-8/CXCL8 and IL-12p70 and the regulatory cytokine IL-10. We analyzed the first two cytokines, as they represent the main cytokines involved in T-cell recruitment and Th1-immune response polarization [31-34] and IL-10 for its suppressive activity on T-cell proliferation and cytokine production [35,36].

In three independent experiments using mDCs from donors 1,2 , and 3, we observed that CTLA-4 engagement by the agonistic mAb significantly reduced IL-8/CXCL8 production by an average of $79.8 \%$ at 24 hours ( $p=0.001, p_{c}=0.007$ ), whereas significativity at 48 hour was lost after Bonferroni correction $\left(p=0.047, p_{c}=0.28\right)$. CTLA-4 engagement also decreased the production of IL-12p70, but this reduction did not reach statistical significance at both 24 and 48 hours ( $p=0.024, p_{c}=0.144$ and $p=0.077, p_{c}=0.462$, respectively) (Fig. 2A). At the same time, CTLA-4 engagement significantly in- 
Table 1

CTLA-4 surface expression on freshly isolated monocytes, monocyte derived immature DCs and mature DCs by flow cytometry ${ }^{\mathrm{a}}$

\begin{tabular}{|c|c|c|c|}
\hline \multirow[t]{4}{*}{ Donor } & \multicolumn{3}{|c|}{ CTLA-4 expression (MRFI) } \\
\hline & \multicolumn{3}{|l|}{$\underline{p_{c}}=0.222^{c}$} \\
\hline & \multirow{2}{*}{$\frac{p_{c}=0.048}{\text { Monocytes }}$} & \multicolumn{2}{|c|}{$\underline{p_{c}=0.002}$} \\
\hline & & Immature DCs & Mature DCs \\
\hline Donor 1 & 5.50 & 1.20 & 2.85 \\
\hline Donor 2 & 4.30 & 1.90 & 2.30 \\
\hline Donor 3 & 4.60 & 1.46 & 2.25 \\
\hline Donor 4 & 4.90 & 2.10 & 3.80 \\
\hline Donor 5 & 2.10 & 2.50 & 3.60 \\
\hline Donor 6 & 6.50 & 0.80 & 1.68 \\
\hline Donor 7 & ND & 0.93 & 2.10 \\
\hline Means & $4.28 \pm 0.62$ & $1.55 \pm 0.24$ & $2.65 \pm 0.30$ \\
\hline
\end{tabular}

${ }^{a}$ CTLA-4 surface expression was evaluated by flow cytometry on freshly isolated human peripheral blood monocytes, immature DCs and LPS-matured DCs, generated from seven independent donors (1-7). Cell samples were stained, by direct immunofluorescence, with the FITC-conjugated anti-CTLA-4 mAb or the isotype control mAb.

${ }^{b}$ Numbers represent mean ratio of relative fluorescence intensity (MRFI), calculated as described in Subjects and methods.

${ }^{\mathrm{c}}$ Differences in CTLA-4 expression among $\mathrm{mDC}$, iDC, and monocytes were evaluated by a two-tailed Student's $t$ test with a $p$ value (significance level $<0.05$ ) further adjusted with Bonferroni correction for multiple comparisons $\left(p_{c}\right)$.

creased IL-10 secretion by an average of $47.2 \%$ at 48 hours ( $p=$ $\left.0.001, p_{c}=0.008\right)$, whereas significativity at 24 hours was not retained after Bonferroni correction ( $p=0.021, p_{c}=0.126$ ) (Fig. $2 \mathrm{~A})$. The cytokine levels produced by anti-CTLA-4-treated mDCs were compared with those produced by mDCs treated with the isotype-matched control $\mathrm{mAb}$. These results suggest a functional role of CTLA-4 signaling pathway in the regulation of cytokine secretion by human mDCs.

The inhibition of IL-8/CXCL8 and IL-12p70 cytokine production was not due to alteration of DC viability, nor to mDC apoptosis, as determined by cytofluorimetry using annexin $\mathrm{V}$ staining and TOPRO-3 uptake, at 24 and 48 hour of culture (Fig. 2B).

These results indicate that CTLA- 4 signal in MDCs can modulate cytokine secretion without interfering with viability and phenotypic maturation.

\subsection{CTLA-4 engagement downregulates the ability of human mature $D C$ s to stimulate Ag-specific CD $4^{+} \mathrm{T}$-cell proliferation}

We next investigated whether CTLA-4 engagement by the agonistic anti-CTLA-4 mAb 3D5 could affect the T-cell proliferationstimulating ability of mDCs. Because CTLA-4 also inhibits T-cell function [1-3], to prevent a direct effect of CTLA-4 mAb on $\mathrm{T}$ lymphocytes, mDCs from three independent donors were pretreated on plates coated with anti-CTLA- 4 mAb or isotypematched control $\mathrm{mAb}$, washed to remove unbound $\mathrm{mAb}$, pulsed with PPD recall antigen, and then added to purified autologous $\mathrm{CD}^{+}{ }^{+} \mathrm{T}$ cells as specified in Subjects and methods. We observed that anti-CTLA-4 pretreated-mDCs significantly reduced the proliferation of $\mathrm{CD}^{+} \mathrm{T}$ cells by $42 \%$ compared with $\mathrm{mDC}$ s treated with the isotype-matched control $\mathrm{mAb}\left(p=0.017, p_{\mathrm{c}}=0.034\right)$ (Fig. 3).

Remarkably, CTLA-4 engagement by the agonistic mAb 3D5 did not affect the expression of DC maturation markers by human LPS-matured DCs as the expression of HLA-DR, CD83, CD80, and CD86 was similar in mDCs cultured, with or without the antiCTLA- 4 mAb, at either 24 or 48 hours. Similarly, CTLA- 4 binding did not affect the expression of either programmed death ligand PDL-1 or PDL-2 (data not shown).

As we observed that IL-10 production by mDCs was increased upon CTLA-4 engagement by the agonistic mAb 3D5, we investigated whether the addition of an anti-IL-10 neutralizing $\mathrm{mAb}$, to PPD loaded DC-T cell co-cultures, could restore T-cell proliferation. The results showed that addition of an anti-IL-10 mAb partially restored the proliferation of antigen-specific $\mathrm{CD}^{+}{ }^{+} \mathrm{T}$ cells cultured with anti-CTLA-4-pretreated-mDCs compared with the culture
A

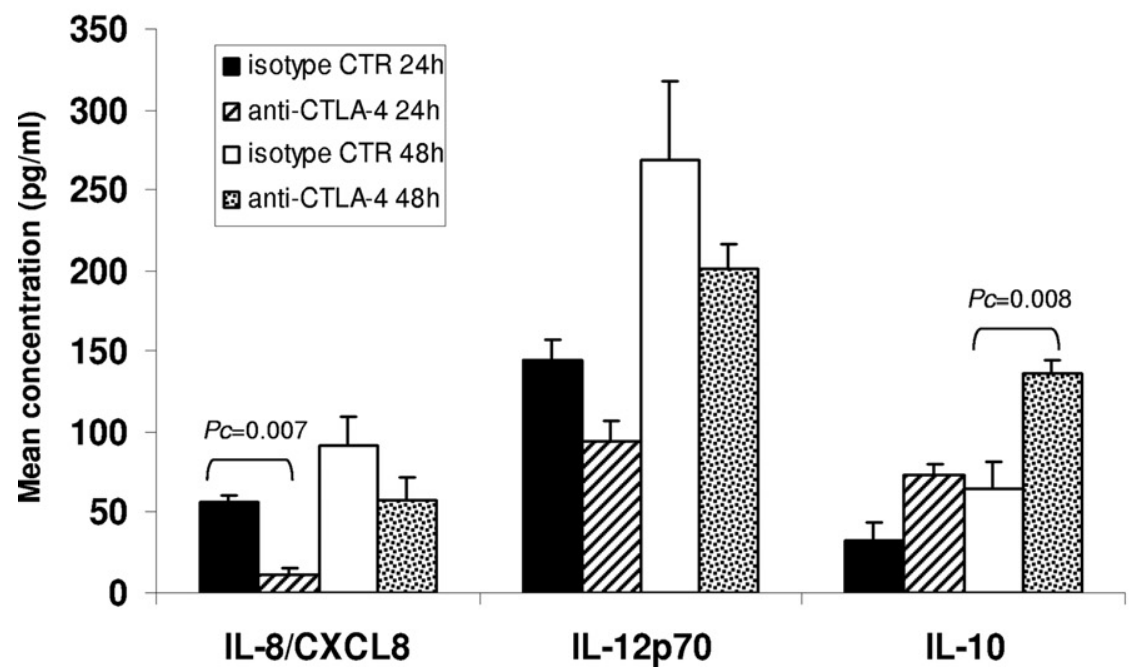

B

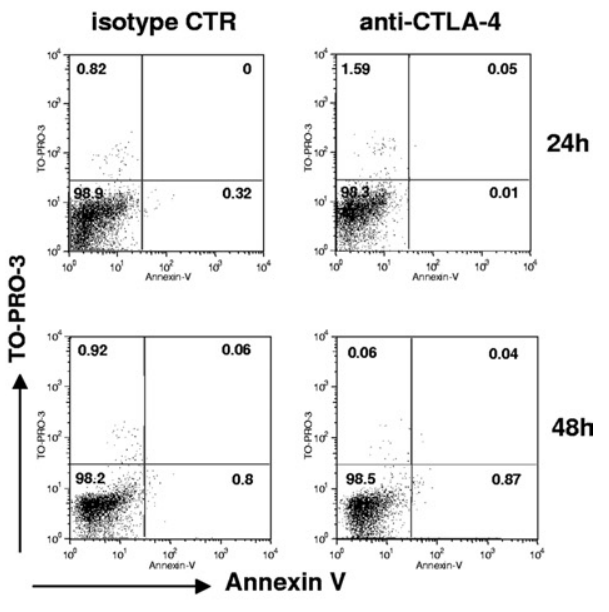

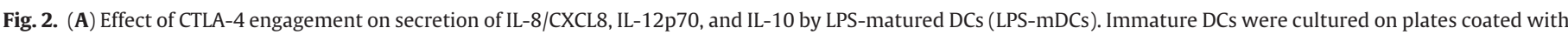

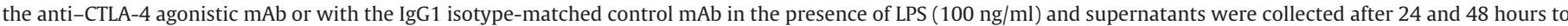

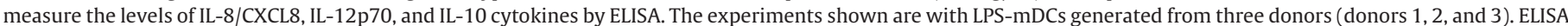

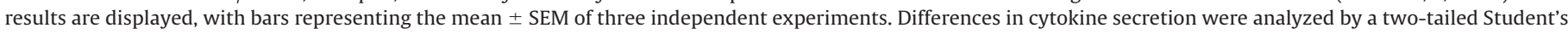

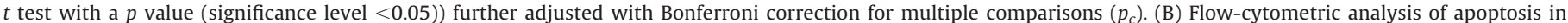

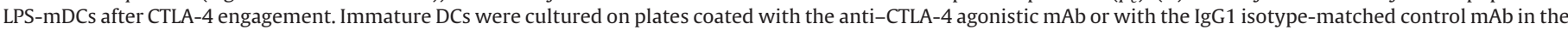

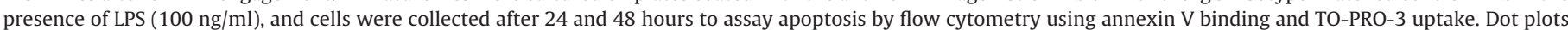

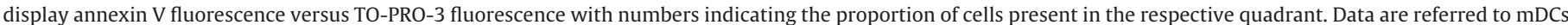
from one representative of the three donors analyzed, whose cytokine production is reported in panel A. 


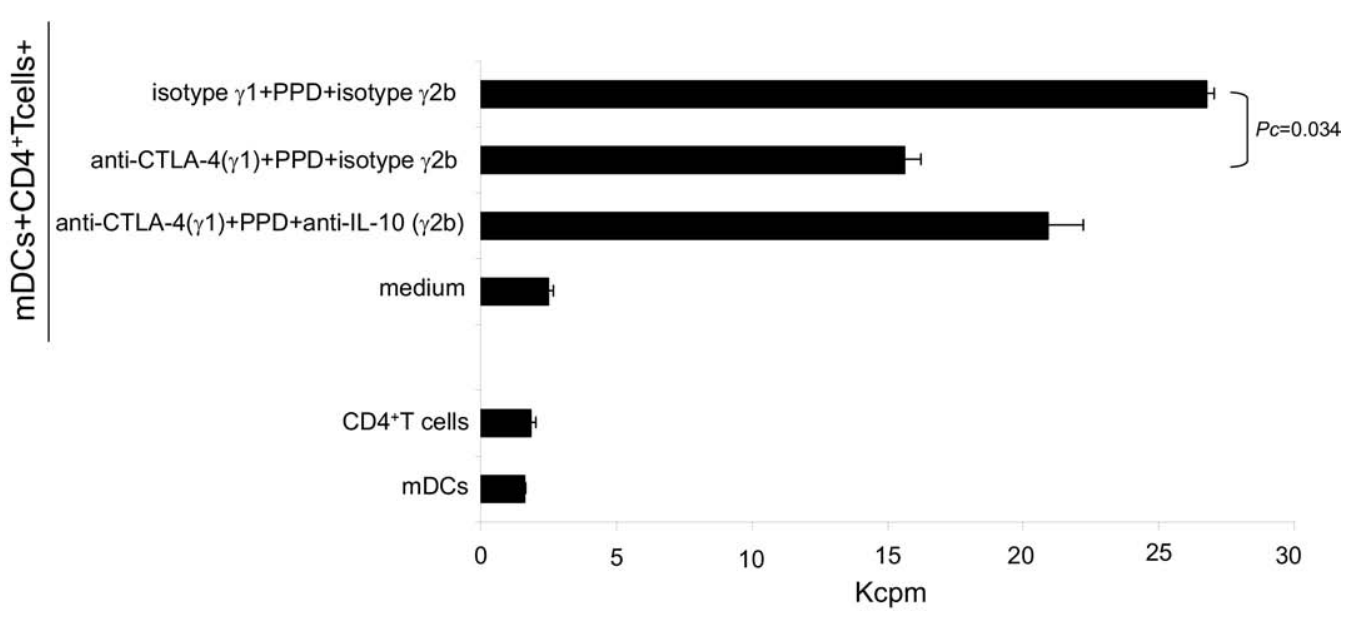

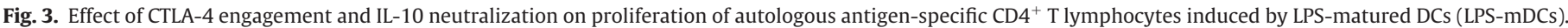

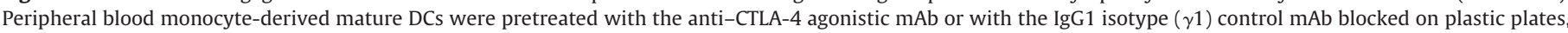

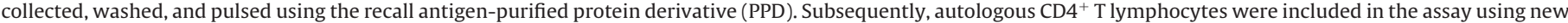

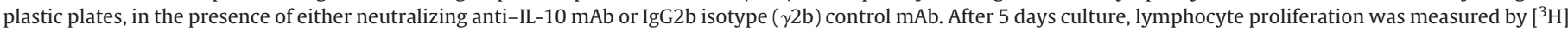

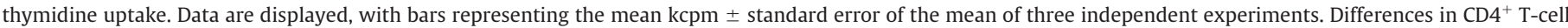

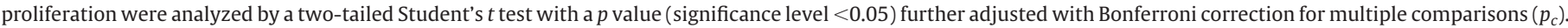

with mDCs treated with anti-CTLA-4 mAb and an irrelevant control $\mathrm{mAb}$ having the same isotype (IgG2b) of the anti-IL-10 mAb (Fig. 3). Although the increase in cell proliferation (25\%) did not reach the statistical significance $\left(p=0.083, p_{c}=0.166\right)$, these data indicate a trend towards restoring $\mathrm{CD} 4^{+} \mathrm{T}$-cell proliferation by IL-10 neutralization. These results indicate that an increased release of IL-10 after CTLA-4-binding of mDCs may be involved in the impairment of antigen-specific T-cell proliferation induced by LPS-matured DCs.

\section{Discussion}

Expression and function of CTLA-4 inhibitory receptor have been investigated mainly in T lymphocytes [15], but recent observations have extended its negative regulatory role to other cell types of the immune system $[5,6]$. In this study we demonstrate, at a protein and transcriptional level, that CTLA-4 receptor is expressed by human monocyte-derived mDCs. We also show that CTLA-4 triggering on mDCs increases IL-10 and reduces IL-8/CXCL8 and IL-12p70 cytokine production, as well as T-cell antigeninduced proliferation.

Our data show that CTLA- 4 is expressed by mature DCs obtained with different stimulations (LPS, Poly:IC, cytokines), whereas it is minimally, or not at all, expressed by immature DCs.

During kinetics studies of DC maturation, we observed that freshly isolated monocytes constitutively express CTLA-4. This observation confirms previous data [6], although we detected higher expression levels, probably because of the use of different antiCTLA- 4 mAbs. This basal expression of CTLA- 4 on monocytes might avoid excessive cell activation and thus sustain immunologic homeostasis.

Interestingly, CTLA-4 expressed by circulating monocytes is rapidly down-modulated upon in vitro culture with GM-CSF+IL-4, but significantly increases as they differentiate into mature DCs in response to stimulation with LPS, PolyI:C, or cytokine cocktail. A similar down-modulation induced by in vitro culture of circulating human monocytes has been described for the expression of phospholipase D (PLD) enzyme which, in turn, is strongly upregulated by LPS [37]. Evidence for a functional link between PLD and CTLA-4 has been shown in T cells in which expression of CTLA-4 at the plasma membrane is caused by CTLA- 4 exocytosis from intracellular vesicles followed by rapid endocytosis [38]. It is noteworthy that the exocytosis pathway of CTLA- 4 in T cells is dependent on the activity of PLD in addition to that of GTPase ADP ribosylation factor-1 [39].

To investigate the functional significance of CTLA-4 expressed by mDCs, we analyzed the effect of CTLA- 4 binding on DC phenotype and functions.

Our results demonstrate that binding of the agonistic antiCTLA- 4 mAb to CTLA-4 expressed by mDCs does not affect the expression of CD83 and HLA-DR, as well as that of the main costimulatory molecules CD80 and CD86. By contrast, under the same experimental conditions, it modulates cytokine secretion.

CTLA-4 engagement induced a marked down-modulating effect on IL-8/CXCL8 production, which remained significant after Bonferroni correction, whereas it showed only a trend toward a significant reduction for IL-12p70 release by mDCs. However, this latter finding may be of relevance as reflecting a biologic phenomenon that might reach statistical significance in larger experimental DC studies. IL-8 is a member of the CXC chemokine family (CXCL8) and plays an important role as a chemotactic factor for all known types of migratory immune cells [31,32]. IL-12p70 heterodimer is the major cytokine promoting TH1-cell-mediated immunity, by inducing T-cell activation, cell proliferation, and production of cytokines, such as IL- 2 and IFN- $\gamma$ [33]. Thus, CTLA- 4 signal in mDCs, by reducing pro-inflammatory and chemoattractant factors such as IL-8 and IL-12, could play an active role in modulating the immune response. DCs can also produce IL-10, which downregulates their own function as well as the ability to stimulate $\mathrm{CD} 4^{+} \mathrm{T}$-cell proliferation [40]. IL-10 is a pleiotropic cytokine that controls inflammatory processes by suppressing the production of pro-inflammatory cytokines that are known to be transcriptionally controlled by NF- $\kappa \mathrm{B}$, including IL-8 and IL-12 [41]. As IL-10 has been found to inhibit the activity of NF- $\kappa \mathrm{B}$ in various cell types, including monocytes/macrophages [42], the inhibitory effect observed on IL-8/ IL-12 cytokine production could be mediated through the IL-10 ability to inhibit NF- $\kappa$ B. IL-10 produced by mDCs was indeed significantly upregulated upon CTLA-4 binding.

Another possible explanation for the inhibitory effect on cytokine production could be a direct blocking of NF- $\kappa$ B or AP- 1 transcription factors that has been reported to occur in activated $T$ cells upon CTLA-4 ligation [6,43,44]. Thus, the inhibitory effect of CTLA-4 binding on pro-inflammatory cytokine secretion by $\mathrm{mDCs}$ is in agreement and extends previous reports on activated $\mathrm{T}$ cells $[14,16]$, as well as B cells [5]. 
In addition, CTLA-4 binding down-modulates CD4 ${ }^{+}$T-cell proliferation induced by LPS-matured DCs in response to the recall antigen PPD. Among the possible mechanisms underlying this finding, the PD-1 T-cell inhibitory signal has been proposed [45]; however we do not favor this hypothesis because, in our case, CTLA-4 binding did not affect PDL-1/PDL-2 expression on mDCs (data not shown). This observation raised the possibility that IL-10 upregulation, upon CTLA-4 engagement, may contribute to the inhibitory effect of PPD-specific CD $4^{+} \mathrm{T}$-cell proliferation in our experimental model. In this regard, IL-10 has been reported to inhibit antigeninduced proliferation and cytokine synthesis by T cells, most probably through its effects on antigen-presenting cells [46]. However, it is likely that IL-10 synergizes with other factors in reducing T-cell proliferation because the addition of neutralizing anti-IL-10 mAb only partially restored $\mathrm{mDC}$-induced $\mathrm{T}$-cell proliferation in response to PPD.

Thus, the CTLA-4 signal might be important in downregulating the DC function by modulating both secretion of pro-inflammatory and anti-inflammatory cytokines as well as DC-induced T-cell response. In contrast, CTLA-4 signal probably does not affect the antigen presenting function of $\mathrm{mDCs}$ as the expression of costimulatory molecules CD80 and CD86 was not altered by CTLA-4 engagement.

Furthermore, binding of CTLA- 4 expressed by mDCs did not affect DC viability as indicated by annexin V/TO-PRO-3 staining, suggesting that the observed inhibitory effects of CTLA- 4 are not a consequence of reduced viability of mDCs.

The expression of other inhibitory receptors belonging, like CTLA-4, to the same immunoglobulin superfamily (IgSF), has been described on human and murine DCs. They include the immunoglobulin-like transcript receptors (ILT2, ILT3, ILT4) [47], which can negatively regulate DC antigen presentation, T-cell costimulation, and cytokine production [48]; the leukocyte Ig-like receptor (LILRB1), which can decrease IL-12 production and DCinduced T-cell response [49]; and the DC-derived immunoglobulin receptor 2 (DIGR2), which mediates negative DC signaling both in vitro and in vivo [50].

Unlike these DC inhibitory receptors, CTLA-4 does not contain a canonical immunotyrosine-based inhibitory motif (ITIM) [51] in the cytoplasmic tail, which is responsible for the transduction of the inhibitory signaling in DC receptors through the recruitment of SHP1 tyrosine phosphatase [52]. Therefore, the mode of action of CTLA-4 may be indirect, thus differing from the other conventional ITIM-containing DC inhibitory receptors.

The expression of CTLA-4 on dendritic cells, to the best of our knowledge not yet reported, is in line with the functional role of CTLA-4 in other CTLA-4-expressing cells studied to date. Therefore, our findings suggest a more general role of this molecule as an attenuator in the immune cell activation not restricted only to $\mathrm{T}$ and B cells. In this respect, the upregulation of CTLA- 4 expressed by matured DCs might be important as a negative feedback mechanism to prevent the excessive activation of T cells. We propose that this regulatory mechanism could result from the signaling delivered by CTLA- 4 to mDCs upon engagement with B7 molecules expressed by $\mathrm{mDC}$ s themselves, or alternatively, with $\mathrm{B} 7$ molecules expressed by activated $\mathrm{T}$ cells. In addition, as B7 molecules expressed by T cells $[53,54]$ are capable of transducing an inhibitory signal to T cells [53], interaction of B7, expressed on T cells, with CTLA-4, expressed by mDCs either in its CTLA-4TM or delTM isoforms, might represent an additional mechanism to terminate Tcell responses [53,55]. Further biochemical and functional characterizations of CTLA- 4 signaling pathway in MDCs will be required to explore this hypothesis.

\section{Acknowledgments}

This work was supported by grants awarded by Ministero dell'Istruzione, dell'Università e della Ricerca (MIUR-PRIN 2007, project WEYB3A) and by Ricerca Sanitaria Regione Liguria (to M.P.P.); by grants from Fondazione CARIGE and Progetti di Ateneo 2007, University of Genova (to D.S.); and by grants from Associazione Italiana per la Ricerca sul Cancro and Ministero della Salute (to G.F.) P.C. was supported by a fellowship from Fondazione Italiana per la Ricerca sul Cancro. The authors thank the blood donors and staff of the Transfusion Center, Galliera Hospital, Genoa, Italy, for kindly providing buffy coats. The authors also thank Simona Pedemonte for the DNA-sequencing service.

\section{References}

[1] Walunas TL, Lenschow DJ, Bakker CY, Linsley PS, Freeman GJ, Green JM, et al. CTLA- 4 can function as a negative regulator of T-cell activation. Immunity 1994; 1:405-13.

[2] Thompson CB, Allison JP. The emerging role of CTLA-4 as an immune attenuator. Immunity 1997; 7:445-50.

[3] Lee KM, Chuang E, Griffin M, Khattri R, Hong DK, Zhang W, et al. Molecular basis of T-cell inactivation by CTLA-4. Science 1998;282:2263-6.

[4] Jonuleit H, Schmitt E, Stassen M, Tuettenberg A, Knop J, Enk AH. Identification and functional characterization of human CD4(+)CD25(+) T cells with regulatory properties isolated from peripheral blood. J Exp Med 2001; 193:1285-94.

[5] Merlo A, Tenca C, Fais F, Battini L, Ciccone E, Grossi CE, Saverino D. Inhibitory receptors CD85j, LAIR-1, and CD152 down-regulate immunoglobulin and cytokine production by human B lymphocytes. Clin Diagn Lab Immunol 2005; 12 : 705-12.

[6] Wang XB, Giscombe R, Yan Z, Heiden T, Xu D, Lefvert AK. Expression of CTLA-4 by human monocytes. Scand J Immunol 2002;55:53-60.

[7] Kaufman KA, Bowen JA, Tsai AF, Bluestone JA, Hunt JS, Ober C. The CTLA-4 gene is expressed in placental fibroblasts. Mol Hum Reprod 1999;5:84-7.

[8] Nagaraju K, Raben N, Villalba ML, Danning C, Loeffler L, Lee E, et al. Costimulatory markers in muscle of patients with idiopathic inflammatory myopathies and in cultured muscle cells. Clin Immunol 1999;92:161-9.

[9] Pistillo MP, Tazzari PL, Palmisano GL, Pierri I, Bolognesi A, Ferlito F, et al. CTLA-4 is not restricted to the lymphoid cell lineage and can function as a target molecule for apoptosis induction of leukemic cells. Blood 2003;101:202-9.

[10] Contardi E, Palmisano GL, Tazzari PL, Martelli AM, Falà F, Fabbi M, et al. CTLA-4 is constitutively expressed on tumor cells and can trigger apoptosis upon ligand interaction. Int J Cancer 2005; 117:538-50.

[11] Shah KV, Chien AJ, Yee C, Moon RT. CTLA-4 is a direct target of Wnt/betacatenin signaling and is expressed in human melanoma tumors. J Invest Dermatol 2008;128:2870-9.

[12] Linsley PS, Greene JL, Brady W, Bajorath J, Ledbetter JA, Peach R. Human B7-1 (CD80) and B7-2 (CD86) bind with similar avidities but distinct kinetics to CD28 and CTLA-4 receptors. Immunity 1994;1:793-801.

[13] Krummel MF, Allison JP. CTLA-4 engagement inhibits IL-2 accumulation and cell cycle progression upon activation of resting T cells. J Exp Med 1996;183: 2533-40.

[14] Brunner MC, Chambers CA, Chan FK, Hanke J, Winoto A, Allison JP. CTLA-4mediated inhibition of early events of T-cell proliferation. J Immunol 1999; 162:5813-20.

[15] Teft WA, Kirchhof MG, Madrenas J. A molecular perspective of CTLA-4 function. Annu Rev Immunol 2006;24:65-97.

[16] Blair PJ, Riley JL, Levine BL, Lee KP, Craighead N, Francomano T, et al. CTLA-4 binding delivers a unique signal to resting human CD4 T cells that inhibits interleukin-2 secretion but allows Bcl-X(L) induction. J Immunol 1998;160: 12-5.

[17] Alegre ML, Shiels H, Thompson CB, Gajewski TF. Expression and function of CTLA-4 in Th1 and Th2 cells. J Immunol 1998;161:3347-56.

[18] Saverino D, Merlo A, Bruno S, Pistoia V, Grossi CE, Ciccone E. Dual effect of CD85/leukocyte Ig-like receptor-1/Ig-like transcript 2 and CD152 (CTLA-4) on cytokine production by antigen-stimulated human T cells. J Immunol 2002; 168:207-15.

[19] Grohmann U, Orabona C, Fallarino F, Vacca C, Calcinaro F, Falorni A, et al. CTLA-4-Ig regulates tryptophan catabolism in vivo. Nat Immunol 2002;3: 1097-101.

[20] Fallarino F, Grohmann U, Hwang KW, Orabona C, Vacca C, Bianchi R, et al. Modulation of tryptophan catabolism by regulatory $\mathrm{T}$ cells. Nat Immunol 2003; 4:1206-12.

[21] Hwu P, Du MX, Lapointe R, Do M, Taylor MW, Young HA. Indoleamine 2,3dioxygenase production by human dendritic cells results in the inhibition of T-cell proliferation. J Immunol 2000;164:3596-9.

[22] Taylor PA, Lees CJ, Fournier S, Allison JP, Sharpe AH, Blazar. BR. B7 expression on T cells down-regulates immune responses through CTLA- 4 binding via T-T interactions. J Immunol 2004;172:34-9.

[23] Paust S, Lu L, McCarty N, Cantor H. Engagement of B7 on effector T cells by regulatory T cells prevents autoimmune disease. Proc Natl Acad Sci U S A 2004;101:10398-403. 
[24] Flores-Borja F, Jury EC, Mauri C, Ehrenstein MR. Defects in CTLA-4 are associated with abnormal regulatory T-cell function in rheumatoid arthritis. Proc Natl Acad Sci U S A 2008;105:19396-401

[25] Sun T, Zhou Y, Yang M, Hu Z, Tan W, Han X, et al. Functional genetic variations in cytotoxic T-lymphocyte antigen 4 and susceptibility to multiple types of cancer. Cancer Res 2008;68:7025-34.

[26] Peggs KS, Quezada SA, Chambers CA, Korman AJ, Allison JP. Blockade of CTLA-4 on both effector and regulatory T-cell compartments contributes to the antitumor activity of anti-CTLA-4 antibodies. J Exp Med 2009;206:1717-25.

[27] Lages CS, Suffia I, Velilla PA. Functional regulatory T cells accumulate in aged hosts and promote chronic infectious disease reactivation. J Immunol 2008; 181:1835-48.

[28] Tenca C, Merlo A, Merck E, Bates EE, Saverino D, Simone R, et al. CD85j (leukocyte Ig-like receptor-1/Ig-like transcript 2) inhibits human osteoclastassociated receptor-mediated activation of human dendritic cells. J Immunol 2005; $174: 6757-63$.

[29] Leung HT, Bradshaw J, Cleaveland JS, Linsley PS. Cytotoxic T lymphocyteassociated molecule-4, a high-avidity receptor for CD80 and CD86, contains an intracellular localization motif in its cytoplasmic tail. J Biol Chem 1995;270: 25107-14.

[30] Schneider H, Martin M, Agarraberes FA, Yin L, Rapoport I, Kirchhausen T, Rudd CE. Cytolytic T lymphocyte-associated antigen- 4 and the TCR $\xi / C D 3$ complex, but not CD28, interact with clathrin adaptor complexes AP-1 and AP-2. J Immunol 1999;163:1868-79.

[31] Taub DD, Anver M, Oppenheim JJ, Longo DL, Murphy WJ. T lymphocyte recruitment by interleukin-8 (IL-8). IL-8-induced degranulation of neutrophils releases potent chemoattractants for human T lymphocytes both in vitro and in vivo. J Clin Invest;1996 1931-41.

[32] Lane HC, Anand AR, Ganju RK. Cbl and Akt regulate CXCL8-induced and CXCR1and CXCR2-mediated chemotaxis. Int Immunol 2006;18:1315-25.

[33] Lanzavecchia A, Sallusto F. Regulation of T-cell immunity by dendritic cells. Cell 2001;106:263-6.

[34] Trinchieri G, Scott P. The role of interleukin 12 in the immune response, disease and therapy. Immunol Today 1994;15:460-3.

[35] Moore KW, O'Garra A, De Waal Malefyt R, Vieira P, Mosmann TR. Interleukin10. Annu Rev Immunol 1993;11:165-90.

[36] Taylor A, Verhagen J, Akkoç T, Wenig R, Flory E, Blaser K, et al. IL-10 suppresses CD2-mediated T-cell activation via SHP-1. Mol Immunol 2009;46:622-9.

[37] Locati M, Riboldi E, Bonecchi R, Transidico P, Bernasconi S, Haribabu B, et al. Selective induction of phospholipase D1 in pathogen-activated human monocytes. Biochem J 2001;358:119-25.

[38] Valk E, Rudd CE, Schneider H. CTLA-4 trafficking and surface expression. Trends Immunol 2008;29:272-9.

[39] Mead KI, Zheng Y, Manzotti CN, Perry LC, Liu MK, Burke F, et al. Exocytosis of CTLA-4 is dependent on phospholipase D and ADP ribosylation factor-1 and stimulated during activation of regulatory $T$ cells. J Immunol 2005; 174:4803-11.

[40] De Smedt T, Van Mechelen M, De Becker G, Urbain J, Leo O, Moser M. Effect of interleukin-10 on dendritic cell maturation and function. Eur J Immunol 1997; 27:1229-35
[41] Wang P, Wu P, Siegel MI, Egan RW, Billah MM. Interleukin (IL)-10 inhibits nuclear factor kappa B (NF kappa B) activation in human monocytes. IL-10 and IL-4 suppress cytokine synthesis by different mechanisms. J Biol Chem 1995 270:9558-63.

[42] de Waal Malefyt R, Abrams J, Bennett B, Figdor CG, de Vries JE. Interleukin 10(IL-10) inhibits cytokine synthesis by human monocytes: An autoregulatory role of IL-10 produced by monocytes. J Exp Med 1991;174:1209-20.

[43] Olsson C, Riesbeck K, Dohlsten M, Michaëlsson E. CTLA-4 ligation suppresses CD28-induced NF-kappaB and AP-1 activity in mouse T-cell blasts. J Biol Chem 1999;274:14400-5.

[44] Pioli C, Gatta L, Frasca D, Doria G. Cytotoxic T lymphocyte antigen. 4 (CTLA-4) inhibits CD28-induced IkappaBalpha degradation and RelA activation. Eur J Immunol 1999;29:856-63.

[45] Freeman GJ, Long AJ, Iwai Y, Bourque K, Chernova T, Nishimura H, et al. Engagement of the PD-1 Immunoinhibitory receptor by a novel B7 family member leads to negative regulation of lymphocyte activation. J Exp Med 2000;192:1027-34.

[46] de Waal Malefyt R, Haanen J, Spits H, Roncarolo MG, te Velde A, Figdor C, et al. Interleukin 10 (IL-10) and viral IL-10 strongly reduce antigen-specific human T-cell proliferation by diminishing the antigen-presenting capacity of monocytes via downregulation of class II major histocompatibility complex expression. J Exp Med 1991;174:915-24.

[47] Colonna M, Nakajima H, Cella M. A family of inhibitory and activating Ig-like receptors that modulate function of lymphoid and myeloid cells. Semin Immunol 2000;12:121-7.

[48] Chang CC, Ciubotariu R, Manavalan JS, Yuan J, Colovai A, Piazza F, et al. Tolerization of dendritic cells by $\mathrm{T}(\mathrm{S})$ cells: The crucial role of inhibitory receptors ILT3 and ILT4. Nat Immunol 2002;3:237-43.

[49] Young NT, Waller EC, Patel R, Roghanian A, Austyn JM, Trowsdale J. The inhibitory receptor LILRB1 modulates the differentiation and regulatory potential of human dendritic cells. Blood 2008;111:3090-6.

[50] Shi L, Luo K, Xia D, Chen T, Chen G, Jiang Y, et al. DIgR2, dendritic cell-derived immunoglobulin receptor 2 , is one representative of a family of IgSF inhibitory receptors and mediates negative regulation of dendritic cell-initiated antigenspecific T-cell responses. Blood 2006;108:2678-86.

[51] Schneider H, Rudd CE. Tyrosine phosphatase SHP-2 binding to CTLA-4: Absence of direct YVKM/YFIP motif recognition. Biochem Biophys Res Commun 2000;269:279-83.

[52] Bolland S, Ravetch JV. Inhibitory pathways triggered by ITIM-containing receptors. Adv Immunol 1999;72:149-77.

[53] Greenwald RJ, Freeman GJ, Sharpe AH. The B7 family revisited. Annu Rev Immunol 2005;23:515-48.

[54] Ferlazzo G, Semino C, Meta M, Procopio F, Morandi B, Melioli G. T lymphocytes express B7 family molecules following interaction with dendritic cells and acquire bystander costimulatory properties. Eur J Immunol 2002;32:3092101

[55] Newland A, Kireta S, Russ G, Krishnan R. Ovine dendritic cells transduced with an adenoviral CTLA4eEGFP fusion protein construct induce hyporesponsiveness to allostimulation. Immunology 2004;113:310-7. 